\title{
Inclusions in a Single Variable in Ultrametric Spaces and Hyers-Ulam Stability
}

\author{
Magdalena Piszczek \\ Pedagogical University, Podchorążych 2, 30-084 Kraków, Poland \\ Correspondence should be addressed to Magdalena Piszczek; magdap@up.krakow.pl
}

Received 7 August 2013; Accepted 2 September 2013

Academic Editors: G. Dai and F. J. Garcia-Pacheco

Copyright (C) 2013 Magdalena Piszczek. This is an open access article distributed under the Creative Commons Attribution License, which permits unrestricted use, distribution, and reproduction in any medium, provided the original work is properly cited.

We present some properties of set-valued inclusions in a single variable in ultrametric spaces. As a consequence, we obtain stability results for the corresponding functional equations.

\section{Introduction}

A metric space $(X, d)$ is called an ultrametric space (or non-Archimedean metric space), if $d$, called an ultrametric, satisfies the strong triangle inequality

$$
d(x, z) \leq \max \{d(x, y), d(y, z)\} \quad \text { for } x, y, z \in X .
$$

One of the typical ultrametrics is a $p$-adic metric. Let $p$ be a fixed prime. For $n, m \in \mathbb{N}$, we define

$$
d(m, n)= \begin{cases}0 & \text { if } m=n, \\ p^{-r} & \text { if } m \neq n,\end{cases}
$$

where $r$ is the largest nonnegative integer such that $p^{r}$ divides $m-n$. This example is the introduction to the $p$-adic numbers which play the essential role because of their connections with some problem coming from quantum physics, $p$-adic string or superstring (see [1]).

The inequality

$$
\begin{array}{r}
d\left(x_{m}, x_{n}\right) \leq \max \left\{d\left(x_{m}, x_{m-1}\right), \ldots, d\left(x_{n+1}, x_{n}\right)\right\} \\
\text { for } m>n
\end{array}
$$

yields and implies the following lemma.

Lemma 1. A sequence $\left(x_{n}\right)_{n \in \mathbb{N}}$ in an ultrametric space $(X, d)$ is a Cauchy sequence if and only if $\lim _{n \rightarrow \infty} d\left(x_{n+1}, x_{n}\right)=0$.

Let $(X, d)$ be an ultrametric space. The number $\delta(A)=$ $\sup \{d(x, y): x, y \in A\}$ is called the diameter of $A \subset X$. We will denote by $n(X)$ the family of all nonempty subsets of $X$. Moreover, let $b d(X)$ stand for the family of all bounded sets of $n(X)$ let and $b \operatorname{cl}(X)$ denote the family of all closed sets of $b d(X)$. We understand the convergence of sets with respect to the Hausdorff metric $h$ derived from the metric $d$. It is easy to see that $(b \mathrm{cl}(X), h)$ is also an ultrametric space, that is, $h$ satisfies the strong triangle inequality

$$
h(A, C) \leq \max \{h(A, B), h(B, C)\} \quad \text { for } A, B, C \in b \mathrm{cl}(X) \text {. }
$$

We say that $(X,+, d)$ is a complete ultrametric commutative groupoid with 0 , if $(X,+)$ is a commutative groupoid with a neutral element $0,(X, d)$ is a complete ultrametric space and the operation + is continuous with respect to the metric $d$.

From now on, we assume that $K$ is a nonempty set and $(X,+, d)$ is a complete ultrametric commutative groupoid with 0 . For $A, B \in n(X)$, we define

$$
A+B=\{a+b: a \in A, b \in B\} .
$$

The aim of the paper is to obtain some results concerning the following inclusion:

$$
\Psi(F(a(x))) \subset F(x)+G(x),
$$

where $\Psi: X \rightarrow X, F, G: K \rightarrow n(X)$, and $a: K \rightarrow K$ and its generalization in an ultrametric space. In ultrametric spaces, it is possible to get better estimation with weaker assumptions, than in metric spaces. The ideas of proofs are 
based on the ideas in [2]. As a consequence we obtain stability results for the corresponding functional equation $\Psi \circ f \circ$ $a=f$ and its generalization. Some results of the stability of functional equations in non-Archimedean spaces can be found in [3-8].

\section{Main Results}

Theorem 2. Let $F, G: K \rightarrow n(X), 0 \in G(x)$ for all $x \in K$, $\Psi: X \rightarrow X, a: K \rightarrow K, \lambda \in(0,1)$, and

$$
\begin{aligned}
& d(\Psi(x), \Psi(y)) \leq \lambda d(x, y), \quad x, y \in X, \\
& M:=\sup \{\delta(F(x)+G(x)): x \in K\}<\infty, \\
& \Psi(F(a(x))) \subset F(x)+G(x) \quad \text { for } x \in K .
\end{aligned}
$$

Then there exists a unique function $f: K \rightarrow X$ such that $\Psi \circ f \circ a=f$ and

$$
d(f(x), F(x)) \leq M, \quad x \in K .
$$

Proof. Let us fix $x \in K$. Replacing $x$ by $a^{n}(x)$ in (8), we get

$$
\Psi\left(F\left(a^{n+1}(x)\right)\right) \subset F\left(a^{n}(x)\right)+G\left(a^{n}(x)\right),
$$

and as $0 \in G(x)$, we have

$$
F\left(a^{n}(x)\right) \subset F\left(a^{n}(x)\right)+G\left(a^{n}(x)\right)
$$

for $n \in \mathbb{N}_{0}$. Thus,

$$
\begin{aligned}
& h\left(\Psi^{n+1}\left(F\left(a^{n+1}(x)\right)\right), \Psi^{n}\left(F\left(a^{n}(x)\right)\right)\right) \\
& \quad \leq \lambda^{n} h\left(\Psi\left(F\left(a^{n+1}(x)\right)\right), F\left(a^{n}(x)\right)\right) \\
& \quad \leq \lambda^{n} \delta\left(F\left(a^{n}(x)\right)+G\left(a^{n}(x)\right)\right) \leq \lambda^{n} M
\end{aligned}
$$

for all $n \in \mathbb{N}_{0}$. According to Lemma 1 and $\lim _{n \rightarrow \infty} \lambda^{n} M=0$, the sequence $\left(\Psi^{n}\left(F\left(a^{n}(x)\right)\right)\right)_{n \in \mathbb{N}_{0}}$ is a Cauchy sequence. Since $(b \mathrm{cl}(Y), h)$ is complete, there exists the limit $\lim _{n \rightarrow \infty} \operatorname{cl} \Psi^{n}\left(F\left(a^{n}(x)\right)\right)$. Moreover,

$$
\delta\left(\operatorname{cl} \Psi^{n}\left(F\left(a^{n}(x)\right)\right)\right) \leq \lambda^{n} \delta\left(F\left(a^{n}(x)\right)\right),
$$

and the right side of the last inequality converges to 0 with $n \rightarrow \infty$. Therefore,

$$
\lim _{n \rightarrow \infty} \operatorname{cl} \Psi^{n}\left(F\left(a^{n}(x)\right)\right)=: f(x)
$$

is a singleton and as $\Psi$ is continuous,

$$
\begin{aligned}
& \Psi(f(a(x))) \\
& \quad=\Psi\left(\lim _{n \rightarrow \infty} \mathrm{cl} \Psi^{n}\left(F\left(a^{n}(a(x))\right)\right)\right) \\
& \quad \subset \lim _{n \rightarrow \infty} \mathrm{cl} \Psi^{n+1}\left(F\left(a^{n+1}(x)\right)\right)=f(x),
\end{aligned}
$$

so $\Psi \circ f \circ a=f$. Notice that

$$
\begin{aligned}
& h\left(\Psi^{n}\left(F\left(a^{n}(x)\right)\right), F(x)\right) \\
& \leq \max \left\{h\left(\Psi^{n}\left(F\left(a^{n}(x)\right)\right), \Psi^{n-1}\left(F\left(a^{n-1}(x)\right)\right)\right), \ldots,\right. \\
& h(\Psi(F(a(x))), F(x))\} \\
& \leq \max \left\{\lambda^{n-1} \delta\left(F\left(a^{n-1}(x)\right)+G\left(a^{n-1}(x)\right)\right), \ldots,\right. \\
& \delta(F(x)+G(x))\} \\
& \leq \max \left\{\lambda^{n-1} M, \ldots, M\right\} \leq M .
\end{aligned}
$$

Consequently,

$$
\begin{gathered}
d(f(x), F(x)) \leq M, \\
f(x) \in F(x)+M S
\end{gathered}
$$

where $S$ is a closed unit ball.

It remains to prove the uniqueness of $f$. Let $f, g$ be such that $\Psi \circ f \circ a=f, \Psi \circ g \circ a=g, d(f(x), F(x)) \leq M$, $d(g(x), F(x)) \leq M$ for $x \in K$. By induction we get $\Psi^{n} \circ f \circ a^{n}=$ $f$ and $\Psi^{n} \circ g \circ a^{n}=g$ for $n \in \mathbb{N}_{0}$. Hence,

$$
\begin{aligned}
d(f(x), g(x)) & =d\left(\Psi^{n} \circ f \circ a^{n}(x), \Psi^{n} \circ g \circ a^{n}(x)\right) \\
& \leq \lambda^{n} d\left(f\left(a^{n}(x)\right), g\left(a^{n}(x)\right)\right) \\
& \leq \lambda^{n} \delta\left(F\left(a^{n}(x)\right)+M S\right), \quad n \in \mathbb{N}_{0} .
\end{aligned}
$$

Since $\lim _{n \rightarrow \infty} \lambda^{n} \delta\left(F\left(a^{n}(x)\right)+M S\right)=0$, we have $f=g$.

Theorem 3. Assume that $F, G: K \rightarrow b d(X), 0 \in G(x)$ for all $x \in K, k \in \mathbb{N}, \Psi: K \times X^{k} \rightarrow X, \alpha_{1}, \ldots, \alpha_{k}: K \rightarrow K$, $\lambda_{1}, \ldots, \lambda_{k}: K \rightarrow[0, \infty)$ are such that

$$
\begin{gathered}
d\left(\Psi\left(x, w_{1}, \ldots, w_{k}\right), \Psi\left(x, z_{1}, \ldots, z_{k}\right)\right) \\
\leq \max _{i \in\{1, \ldots, k\}} \lambda_{i}(x) d\left(w_{i}, z_{i}\right)
\end{gathered}
$$

for $x \in K, w_{1}, \ldots, w_{k}, z_{1}, \ldots, z_{k} \in X$

$$
\begin{aligned}
& \lim _{n \rightarrow \infty} \max _{i_{1}=1, \ldots, k} \lambda_{i_{1}}(x) \max _{i_{2}=1, \ldots, k}\left(\lambda_{i_{2}} \circ \alpha_{i_{1}}\right)(x) \cdots \\
& \max _{i_{n}=1, \ldots, k}\left(\lambda_{i_{n}} \circ \alpha_{i_{n-1}} \circ \cdots \circ \alpha_{i_{1}}\right)(x) \\
& \quad \times \delta\left(F\left(\left(\alpha_{i_{n}} \circ \cdots \circ \alpha_{i_{1}}\right)(x)\right)\right. \\
& \left.\quad+G\left(\left(\alpha_{i_{n}} \circ \cdots \circ \alpha_{i_{1}}\right)(x)\right)\right)=0, \quad x \in K,
\end{aligned}
$$

$$
\begin{aligned}
& \Psi\left(x, F\left(\alpha_{1}(x)\right), \ldots, F\left(\alpha_{k}(x)\right)\right) \\
& \subset F(x)+G(x) \quad \text { for } x \in K .
\end{aligned}
$$


Then there exists a unique function $f: K \rightarrow X$ such that $\Psi\left(x, f\left(\alpha_{1}(x)\right), \ldots, f\left(\alpha_{k}(x)\right)\right)=f(x)$ and $d(f(x), F(x)) \leq$ $k(x)$ for $x \in K$, where

$$
\begin{aligned}
& k(x)=\sup _{n \in \mathbb{N}}\{\delta(F(x)+G(x)), \\
& \max _{i_{1}=1, \ldots, k} \lambda_{i_{1}}(x) \max _{i_{2}=1, \ldots, k}\left(\lambda_{i_{2}} \circ \alpha_{i_{1}}\right)(x) \cdots \\
& \max _{i_{n}=1, \ldots, k}\left(\lambda_{i_{n}} \circ \alpha_{i_{n-1}} \circ \cdots \circ \alpha_{i_{1}}\right)(x) \\
& \times \delta\left(F\left(\left(\alpha_{i_{n}} \circ \cdots \circ \alpha_{i_{1}}\right)(x)\right)\right. \\
&\left.\left.+G\left(\left(\alpha_{i_{n}} \circ \cdots \circ \alpha_{i_{1}}\right)(x)\right)\right)\right\} .
\end{aligned}
$$

Proof. Let us fix $x \in K$. Replacing $x$ by $\alpha_{i}(x), i=1, \ldots, k$, in (21), we obtain

$$
\begin{gathered}
\Psi\left(\alpha_{i}(x), F\left(\alpha_{1}\left(\alpha_{i}(x)\right)\right), \ldots, F\left(\alpha_{k}\left(\alpha_{i}(x)\right)\right)\right) \\
\subset F\left(\alpha_{i}(x)\right)+G\left(\alpha_{i}(x)\right) .
\end{gathered}
$$

Since $0 \in G(x)$, we have $F\left(\alpha_{i}(x)\right) \subset F\left(\alpha_{i}(x)\right)+G\left(\alpha_{i}(x)\right)$ for $i=1, \ldots, k$. Hence,

$$
\begin{gathered}
\Psi\left(x, \Psi\left(\alpha_{1}(x), F\left(\alpha_{1}\left(\alpha_{1}(x)\right)\right), \ldots, F\left(\alpha_{k}\left(\alpha_{1}(x)\right)\right)\right), \ldots,\right. \\
\left.\Psi\left(\alpha_{k}(x), F\left(\alpha_{1}\left(\alpha_{k}(x)\right)\right), \ldots, F\left(\alpha_{k}\left(\alpha_{k}(x)\right)\right)\right)\right) \\
\subset \Psi\left(x, F\left(\alpha_{1}(x)\right)+G\left(\alpha_{1}(x)\right), \ldots, F\left(\alpha_{k}(x)\right)\right. \\
\left.+G\left(\alpha_{k}(x)\right)\right)
\end{gathered}
$$

We define a sequence $\left(A_{n}(x)\right)_{n \in \mathbb{N}_{0}}$ by the following recurrence relation:

$$
\begin{gathered}
A_{0}(x)=F(x), \\
A_{n+1}(x)=\Psi\left(x, A_{n}\left(\alpha_{1}(x)\right), \ldots, A_{n}\left(\alpha_{k}(x)\right)\right) .
\end{gathered}
$$

It is easy to see that

$$
\begin{aligned}
& h\left(A_{1}(x), A_{0}(x)\right) \\
& \leq h\left(\Psi\left(x, F\left(\alpha_{1}(x)\right), \ldots, F\left(\alpha_{k}(x)\right)\right), F(x)\right) \\
& \leq \delta(F(x)+G(x)), \\
& h\left(A_{n+1}(x), A_{n}(x)\right) \\
& \leq \max _{i_{1}=1, \ldots, k} \lambda_{i_{1}}(x) \max _{i_{2}=1, \ldots, k}\left(\lambda_{i_{2}} \circ \alpha_{i_{1}}\right)(x) \ldots \\
& \quad \max _{i_{n}=1, \ldots, k}\left(\lambda_{i_{n}} \circ \alpha_{i_{n-1}} \circ \ldots \circ \alpha_{i_{1}}\right)(x) \\
& \quad \times \delta\left(F\left(\left(\alpha_{i_{n}} \circ \ldots \circ \alpha_{i_{1}}\right)(x)\right)+G\left(\left(\alpha_{i_{n}} \circ \ldots \circ \alpha_{i_{1}}\right)(x)\right)\right)
\end{aligned}
$$

for $n \in \mathbb{N}$. In virtue of $(20)$, the sequence $\left(A_{n}(x)\right)_{n \in \mathbb{N}}$ is a Cauchy sequence. As $(b \operatorname{cl}(Y), h)$ is a complete metric space, there exists the $\operatorname{limit}_{n \rightarrow \infty} \lim A_{n}(x)$. Moreover,

$$
\begin{aligned}
& \delta\left(\operatorname{cl} A_{n}(x)\right) \\
& \leq \max _{i_{1}=1, \ldots, k} \lambda_{i_{1}}(x) \max _{i_{2}=1, \ldots, k}\left(\lambda_{i_{2}} \circ \alpha_{i_{1}}\right)(x) \cdots \\
& \quad \max _{i_{n}=1, \ldots, k}\left(\lambda_{i_{n}} \circ \alpha_{i_{l-1}} \circ \cdots \circ \alpha_{i_{1}}\right)(x) \\
& \quad \times \delta\left(F\left(\left(\alpha_{i_{n}} \circ \ldots \circ \alpha_{i_{1}}\right)(x)\right)\right)
\end{aligned}
$$

and the right side of the last inequality converges to 0 as $n \rightarrow$ $\infty$. Therefore,

$$
\lim _{n \rightarrow \infty} \operatorname{cl} A_{n}(x)=: f(x)
$$

is a singleton, and as $\Psi$ satisfies (19),

$$
\begin{aligned}
\Psi(x, f & \left.\left(\alpha_{1}(x)\right), \ldots, f\left(\alpha_{k}(x)\right)\right) \\
= & \Psi\left(x, \lim _{n \rightarrow \infty} \mathrm{cl} A_{n}\left(\alpha_{1}(x)\right), \ldots, \lim _{n \rightarrow \infty} \mathrm{cl} A_{n}\left(\alpha_{k}(x)\right)\right) \\
& \subset \lim _{n \rightarrow \infty} \mathrm{cl} \Psi\left(x, A_{n}\left(\alpha_{1}(x)\right), \ldots, A_{n}\left(\alpha_{k}(x)\right)\right) \\
= & \lim _{n \rightarrow \infty} \mathrm{cl} A_{n+1}(x)=f(x) .
\end{aligned}
$$

By (26), we get

$$
\begin{aligned}
& h\left(A_{n}(x), F(x)\right) \\
& \leq \max \{\delta(F(x)+G(x)), \ldots, \\
& \max _{i_{1}=1, \ldots, k} \lambda_{i_{1}}(x) \max _{i_{2}=1, \ldots, k}\left(\lambda_{i_{2}} \circ \alpha_{i_{1}}\right)(x) \cdots \\
& \max _{i_{n-1}=1, \ldots, k}\left(\lambda_{i_{n-1}} \circ \alpha_{i_{n-2}} \circ \ldots \circ \alpha_{i_{1}}\right)(x) \\
& \quad \times \delta\left(F\left(\left(\alpha_{i_{n-1}} \circ \ldots \circ \alpha_{i_{1}}\right)(x)\right)\right. \\
& \left.\left.\quad+G\left(\left(\alpha_{i_{n-1}} \circ \ldots \circ \alpha_{i_{1}}\right)(x)\right)\right)\right\}
\end{aligned}
$$

which, with $n \rightarrow \infty$, yields

$$
\begin{aligned}
& d(f(x), F(x)) \leq \sup _{n \in \mathbb{N}}\{\delta(F(x)+G(x)), \\
& \max _{i_{1}=1, \ldots, k} \lambda_{i_{1}}(x) \max _{i_{2}=1, \ldots, k}\left(\lambda_{i_{2}} \circ \alpha_{i_{1}}\right)(x) \cdots \\
& \max _{i_{n}=1, \ldots, k}\left(\lambda_{i_{n}} \circ \alpha_{i_{n-1}} \circ \cdots \circ \alpha_{i_{1}}\right)(x) \\
& \quad \times \delta\left(F\left(\left(\alpha_{i_{n}} \circ \cdots \circ \alpha_{i_{1}}\right)(x)\right)\right. \\
& \left.\left.\quad+G\left(\left(\alpha_{i_{n}} \circ \cdots \circ \alpha_{i_{1}}\right)(x)\right)\right)\right\} \\
& =k(x) .
\end{aligned}
$$


It remains to prove the uniqueness of $f$. Suppose that $f, g$ are such that $\Psi\left(x, f\left(\alpha_{1}(x)\right), \ldots, f\left(\alpha_{k}(x)\right)\right)=f(x), \Psi(x$, $\left.g\left(\alpha_{1}(x)\right), \ldots, g\left(\alpha_{k}(x)\right)\right)=g(x), d(f(x), F(x)) \leq k(x)$, and $d(g(x), F(x)) \leq k(x)$. Replacing $x$ by $\alpha_{i}(x), i=1, \ldots, k$, in the penultimate equality, we get

$$
\Psi\left(\alpha_{i}(x), f\left(\alpha_{1}\left(\alpha_{i}(x)\right)\right), \ldots, f\left(\alpha_{k}\left(\alpha_{i}(x)\right)\right)\right)=f\left(\alpha_{i}(x)\right) .
$$

Thus,

$$
\begin{aligned}
\Psi(x, & \Psi\left(\alpha_{1}(x), f\left(\alpha_{1}\left(\alpha_{1}(x)\right)\right), \ldots, f\left(\alpha_{k}\left(\alpha_{1}(x)\right)\right)\right), \ldots, \\
& \left.\Psi\left(\alpha_{k}(x), f\left(\alpha_{1}\left(\alpha_{k}(x)\right)\right), \ldots, f\left(\alpha_{k}\left(\alpha_{k}(x)\right)\right)\right)\right) \\
& =\Psi\left(x, f\left(\alpha_{1}(x)\right), \ldots, f\left(\alpha_{k}(x)\right)\right)=f(x),
\end{aligned}
$$

and we get a constant sequence

$$
\begin{gathered}
f_{0}(x)=f(x), \\
f_{n+1}(x)=\Psi\left(x, f_{n}\left(\alpha_{1}(x)\right), \ldots, f_{n}\left(\alpha_{k}(x)\right)\right) .
\end{gathered}
$$

In the same way, we get a constant sequence

$$
\begin{gathered}
g_{0}(x)=g(x), \\
g_{n+1}(x)=\Psi\left(x, g_{n}\left(\alpha_{1}(x)\right), \ldots, g_{n}\left(\alpha_{k}(x)\right)\right) .
\end{gathered}
$$

Hence,

$$
\begin{aligned}
d(f(x), g(x))= & d\left(f_{n}(x), g_{n}(x)\right) \\
\leq & \max _{i_{1}=1, \ldots, k} \lambda_{i_{1}}(x) \max _{i_{2}=1, \ldots, k}\left(\lambda_{i_{2}} \circ \alpha_{i_{1}}\right)(x) \ldots \\
& \max _{i_{n}=1, \ldots, k}\left(\lambda_{i_{n}} \circ \alpha_{i_{n-1}} \circ \cdots \circ \alpha_{i_{1}}\right)(x) \\
& \times d\left(f\left(\left(\alpha_{i_{n}} \circ \cdots \circ \alpha_{i_{1}}\right)(x)\right),\right. \\
& \left.g\left(\left(\alpha_{i_{n}} \circ \cdots \circ \alpha_{i_{1}}\right)(x)\right)\right) \\
\leq & \max _{i_{1}=1, \ldots, k} \lambda_{i_{1}}(x) \max _{i_{2}=1, \ldots, k}\left(\lambda_{i_{2}} \circ \alpha_{i_{1}}\right)(x) \ldots \\
& \max _{i_{n}=1, \ldots, k}\left(\lambda_{i_{n}} \circ \alpha_{i_{n-1}} \circ \cdots \circ \alpha_{i_{1}}\right)(x) \\
& \times\left(d \left(f\left(\left(\alpha_{i_{n}} \circ \cdots \circ \alpha_{i_{1}}\right)(x)\right),\right.\right. \\
& \left.\quad F\left(\left(\alpha_{i_{n}} \circ \cdots \circ \alpha_{i_{1}}\right)(x)\right)\right) \\
& \quad+d\left(F\left(\left(\alpha_{i_{n}} \circ \cdots \circ \alpha_{i_{1}}\right)(x)\right),\right. \\
& \left.\left.\quad g\left(\left(\alpha_{i_{n}} \circ \cdots \circ \alpha_{i_{1}}\right)(x)\right)\right)\right) \\
\leq & 2 \max _{i_{1}=1, \ldots, k} \lambda_{i_{1}}(x) \max _{i_{2}=1, \ldots, k}\left(\lambda_{i_{2}} \circ \alpha_{i_{1}}\right)(x) \ldots \\
& \max _{i_{n}=1, \ldots, k}\left(\lambda_{i_{n}} \circ \alpha_{i_{n-1}} \circ \cdots \circ \alpha_{i_{1}}\right)(x) \\
& \times k\left(\left(\alpha_{i_{n}} \circ \cdots \circ \alpha_{i_{1}}\right)(x)\right) .
\end{aligned}
$$

Using the definition of $k$, we get

$$
\begin{aligned}
& d(f(x), g(x)) \leq 2 \max _{i_{1}=1, \ldots, k} \lambda_{i_{1}}(x) \max _{i_{2}=1, \ldots, k}\left(\lambda_{i_{2}} \circ \alpha_{i_{1}}\right)(x) \cdots \\
& \max _{i_{n}=1, \ldots, k}\left(\lambda_{i_{n}} \circ \alpha_{i_{n-1}} \circ \cdots \circ \alpha_{i_{1}}\right)(x) \\
& \times\left(\delta \left(F\left(\left(\alpha_{i_{n}} \circ \cdots \circ \alpha_{i_{1}}\right)(x)\right)\right.\right. \\
& +G\left(\left(\alpha_{i_{n}} \circ \cdots \circ \alpha_{i_{1}}\right)(x)\right) \\
& +\sup _{l=1, \ldots, \infty} \max _{j_{1}=1, \ldots, k}\left(\lambda_{j_{1}} \circ \alpha_{i_{n-1}} \circ \cdots \circ \alpha_{i_{1}}\right) \\
& \times(x) \cdots \max _{j_{l}=1, \ldots, k}\left(\lambda_{j_{l}} \circ \alpha_{j_{l-1}} \circ \cdots \circ \alpha_{j_{1}}\right. \\
& \left.\circ \alpha_{i_{n-1}} \circ \cdots \circ \alpha_{i_{1}}\right)(x) \\
& \times \delta\left(F \left(\left(\alpha_{j_{l}} \circ \cdots \circ \alpha_{j_{1}} \circ \alpha_{i_{n-1}}\right.\right.\right. \\
& \left.\left.\circ \cdots \circ \alpha_{i_{1}}\right)(x)\right) \\
& +G\left(\left(\alpha_{j_{n}} \circ \cdots \circ \alpha_{j_{1}} \circ \alpha_{i_{n-1}}\right.\right. \\
& \left.\left.\left.\left.\circ \ldots \circ \alpha_{i_{1}}(x)\right)\right)\right)\right) \\
& =\sup _{l=n, \ldots, \infty} \max _{i_{1}=1, \ldots, k} \lambda_{i_{1}}(x) \max _{i_{2}=1, \ldots, k}\left(\lambda_{i_{2}} \circ \alpha_{i_{1}}\right) \\
& \times(x) \cdots \max _{i_{l}=1, \ldots, k}\left(\lambda_{i_{l}} \circ \alpha_{i_{l-1}} \circ \cdots \circ \alpha_{i_{1}}\right)(x) \\
& \times \delta\left(F\left(\left(\alpha_{i_{l}} \circ \cdots \circ \alpha_{i_{1}}(x)\right)\right)\right. \\
& \left.+G\left(\left(\alpha_{i_{l}} \circ \cdots \circ \alpha_{i_{1}}\right)(x)\right)\right) \text {. }
\end{aligned}
$$

It follows that $f=g$ with $n \rightarrow 0$, and the proof is completed.

\section{Stability Results}

We present the applications of the above theorems to the results concerning the stability of functional equations.

Corollary 4. Let $f: K \rightarrow X, \Psi: X \rightarrow X, a: K \rightarrow K$, $\lambda \in(0,1), \epsilon>0$ satisfy

$$
\begin{gathered}
d(\Psi(x), \Psi(y)) \leq \lambda d(x, y), \quad x, y \in X, \\
d(\Psi(f(a(x))), f(x)) \leq \epsilon, \quad x \in K .
\end{gathered}
$$

Then there exists a unique function $g: K \rightarrow X$ such that $\Psi \circ g \circ a=g$ and

$$
d(f(x), g(x)) \leq 2 \epsilon, \quad x \in K .
$$

Proof. Let $F(x)=\{f(x)\}$ for $x \in K$. Then

$$
\Psi(F(a(x))) \subset F(x)+\epsilon S,
$$


where $S$ is a closed unit ball and

$$
\delta(F(x)+\epsilon S)=2 \epsilon
$$

According to Theorem 2 there exists a unique function $g$ such that $\Psi \circ g \circ a=g$ and $d(f(x), g(x)) \leq 2 \epsilon$ for $x \in K$.

Corollary 5. Let $f: K \rightarrow X, k \in \mathbb{N}, \alpha_{1}, \ldots, \alpha_{k}: K \rightarrow K$, $\lambda_{1}, \ldots, \lambda_{k}: K \rightarrow[0,1], \epsilon>0, \Psi: K \times X^{k} \rightarrow X$ satisfy (19) and

$$
d\left(\Psi\left(x, f\left(\alpha_{1}(x)\right), \ldots, f\left(\alpha_{k}(x)\right)\right), f(x)\right) \leq \epsilon \quad \text { for } x \in K
$$

Then there exists a unique function $g$ such that

$$
\begin{gathered}
\Psi\left(x, g\left(\alpha_{1}(x)\right), \ldots, g\left(\alpha_{k}(x)\right)\right)=g(x), \\
d(f(x), g(x)) \leq 2 \epsilon .
\end{gathered}
$$

Proof. Let $F(x)=\{f(x)\}$ for $x \in K$. Then

$$
\Psi\left(x, F\left(\alpha_{1}(x)\right), \ldots, F\left(\alpha_{k}(x)\right)\right) \subset F(x)+\epsilon S,
$$

where $S$ is a closed unit ball and

$$
\begin{gathered}
\sup _{l=1, \ldots, \infty}\left\{\delta(F(x)+\epsilon S), \max _{i_{1}=1, \ldots, k} \lambda_{i_{1}}(x) \max _{i_{2}=1, \ldots, k}\left(\lambda_{i_{2}} \circ \alpha_{i_{1}}\right)(x) \cdots\right. \\
\max _{i_{l}=1, \ldots, k}\left(\lambda_{i_{l}} \circ \alpha_{i_{l-1}} \circ \ldots \circ \alpha_{i_{1}}\right)(x) \\
\left.\quad \times \delta\left(F\left(\left(\alpha_{i_{l}} \circ \ldots \circ \alpha_{i_{1}}\right)(x)\right)+\epsilon S\right)\right\}=2 \epsilon .
\end{gathered}
$$

By Theorem 3, we get the assertion.

As it was observed in $[9,10]$ from the stability results concerning the equation $\Psi \circ f \circ a=f$, we can easily derive the stability of functional equations in several variables, for example, the Cauchy equation, the Jensen equation, or the quadratic equation. The equation $\Psi\left(x, f\left(\alpha_{1}(x)\right), \ldots, f\left(\alpha_{k}(x)\right)\right)=f(x)$ is a generalization of the gamma-type equations or the linear equations (see $[11,12]$ ).

\section{References}

[1] A. Khrennikov, Non-Archimedean Analysis: Quantum Paradoxes, Dynamical Systems and Biological Models, vol. 427 of Mathematics and Its Applications, Kluwer Academic Publishers, Dordrecht, The Netherlands, 1997.

[2] M. Piszczek, "The properties of functional inclusions and Hyers-Ulam stability," Aequationes Mathematicae, vol. 85, pp. 111-118, 2013.

[3] K. Ciepliński, "Stability of multi-additive mappings in nonArchimedean normed spaces," Journal of Mathematical Analysis and Applications, vol. 373, no. 2, pp. 376-383, 2011.

[4] Y. J. Cho, C. Park, and R. Saadati, "Functional inequalities in non-Archimedean Banach spaces," Applied Mathematics Letters, vol. 23, no. 10, pp. 1238-1242, 2010.
[5] Z. Kaiser, "On stability of the Cauchy equation in normed spaces over fields with valuation," Publicationes Mathematicae, vol. 64, no. 1-2, pp. 189-200, 2004.

[6] Z. Kaiser, "On stability of the monomial functional equation in normed spaces over fields with valuation," Journal of Mathematical Analysis and Applications, vol. 322, no. 2, pp. 1188-1198, 2006.

[7] M. S. Moslehian and T. M. Rassias, "Stability of functional equations in non-archimedean spaces," Applicable Analysis and Discrete Mathematics, vol. 1, no. 2, pp. 325-334, 2007.

[8] J. Schwaiger, "Functional equations for homogeneous polynomials arising from multilinear mappings and their stability," Annales Mathematicae Silesianae, vol. 8, pp. 157-171, 1994.

[9] J. Brzdęk, "On a method of proving the Hyers-Ulam stability of functional equations on restricted domains," The Australian Journal of Mathematical Analysis and Applications, vol. 6, no. 1, pp. 1-10, 2009.

[10] G.-L. Forti, "Comments on the core of the direct method for proving Hyers-Ulam stability of functional equations," Journal of Mathematical Analysis and Applications, vol. 295, no. 1, pp. 127-133, 2004.

[11] T. Trif, "Hyers-Ulam-Rassias stability of a linear functional equation with constant coeffcients," Nonlinear Functional Analysis and Applications, vol. 11, pp. 881-889, 2006.

[12] T. Trif, "On the stability of a general gamma-type functional equation," Publicationes Mathematicae, vol. 60, no. 1-2, pp. 4761, 2002. 


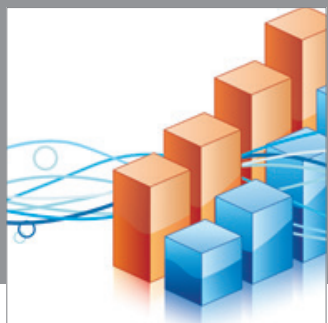

Advances in

Operations Research

mansans

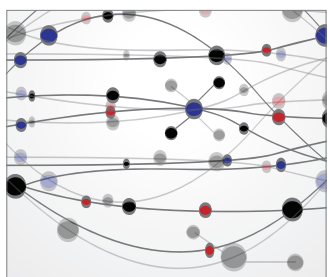

The Scientific World Journal
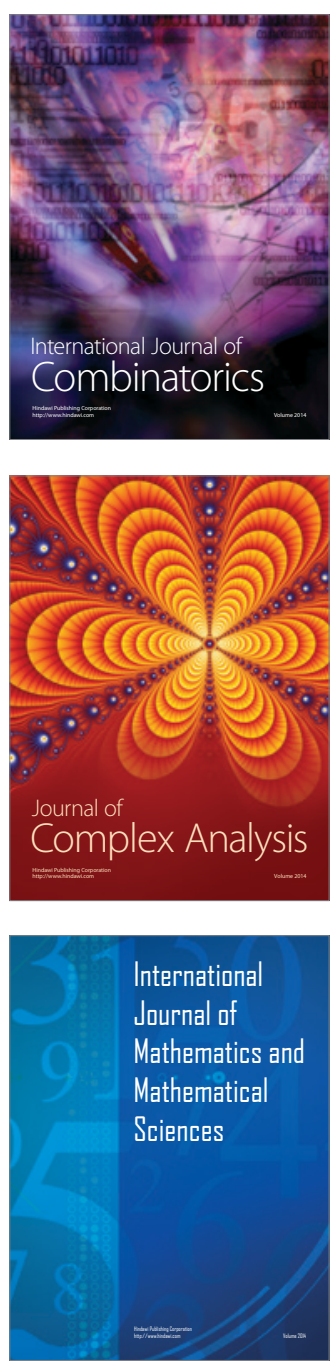
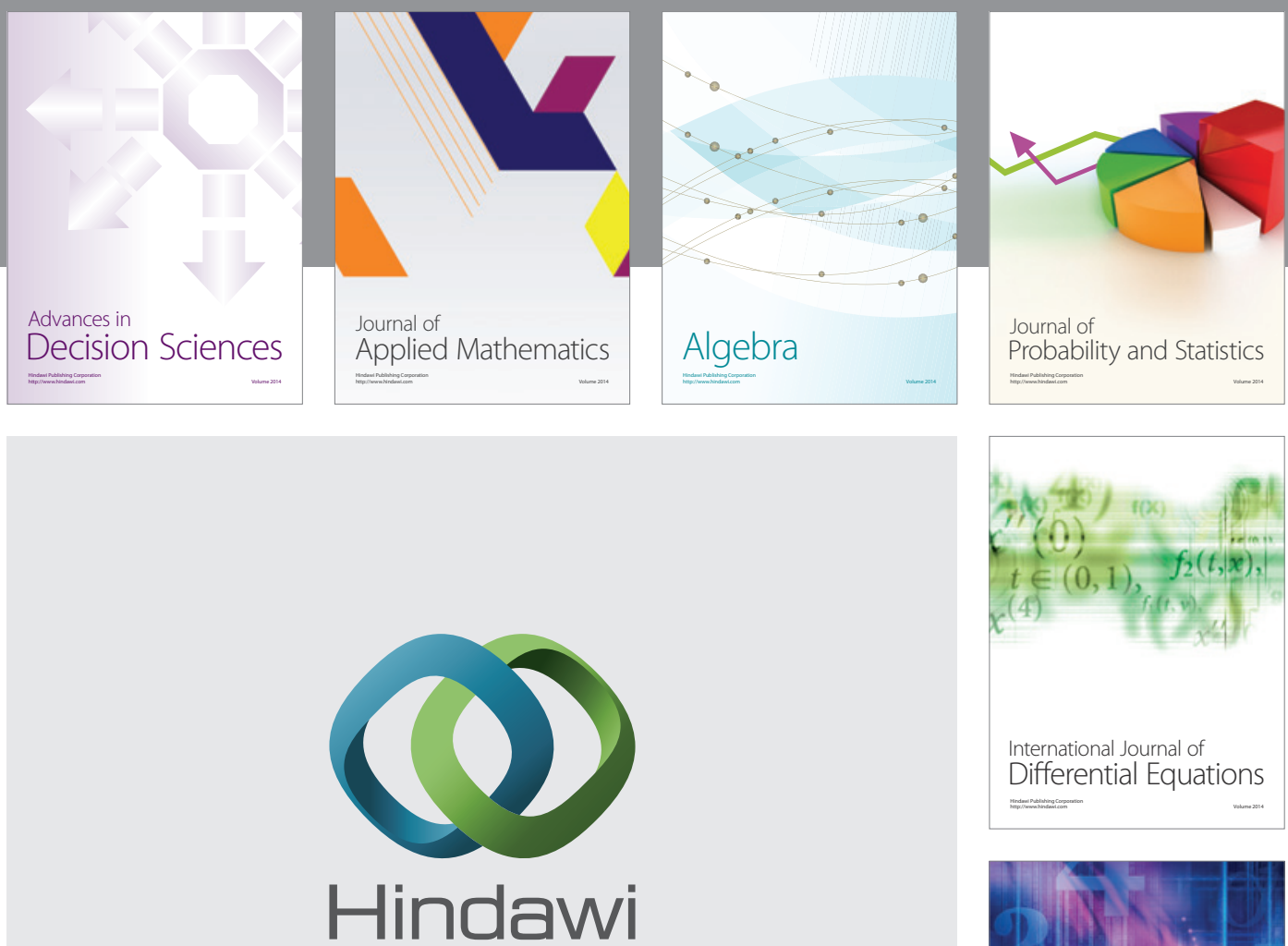

Submit your manuscripts at http://www.hindawi.com
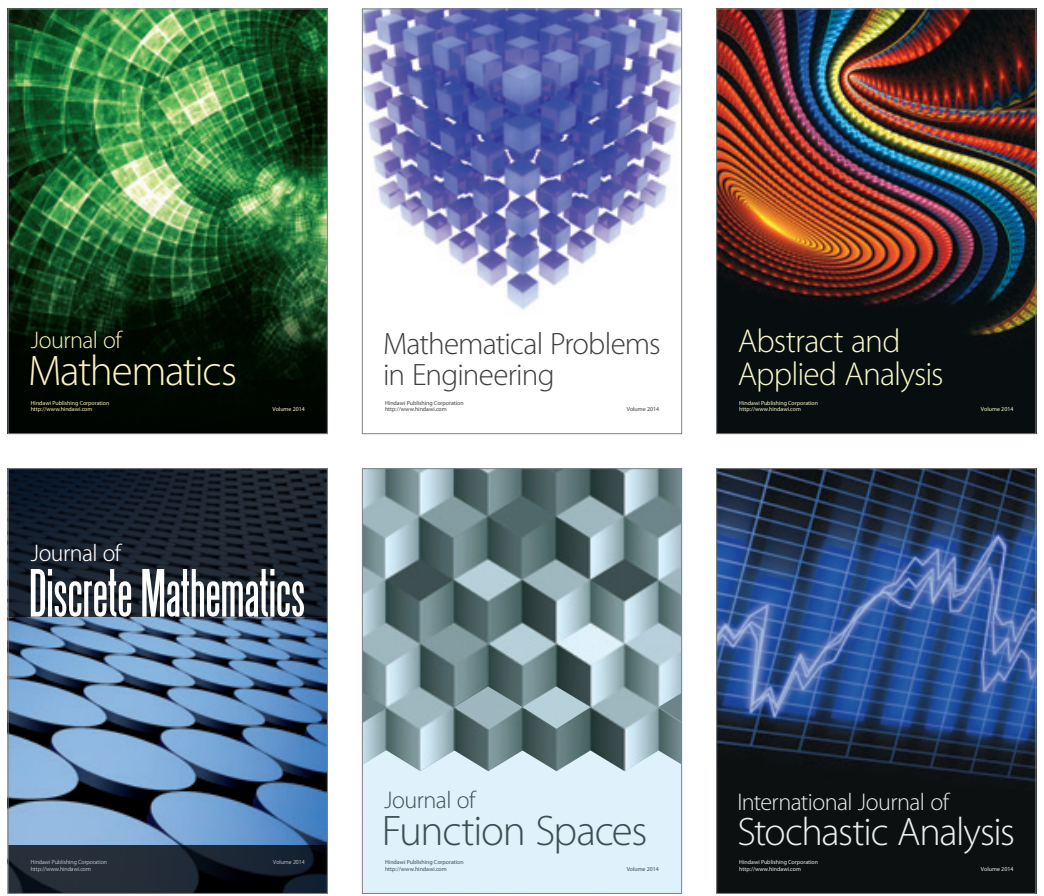

Journal of

Function Spaces

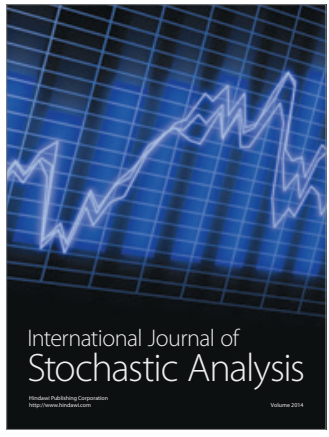

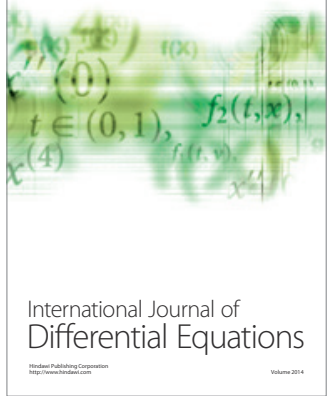
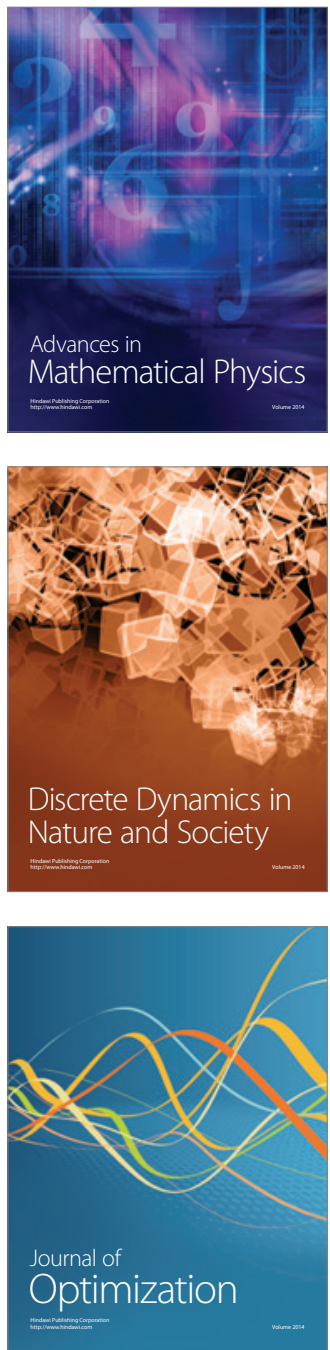